\title{
Intravitreal ranibizumab in treating extensive traumatic submacular hemorrhage
}

This article was published in the following Dove Press journal:

Clinical Ophthalmology

5 April 2013

Number of times this article has been viewed

\author{
Ismail Abdul-Salim ' \\ Zunaina Embong ${ }^{1,2}$ \\ Sonny-Teo Khairy-Shamel ${ }^{1,2}$ \\ Mohd-Noor Raja-Azmi ${ }^{1,2}$ \\ 'Department of Ophthalmology, \\ School of Medical Sciences, \\ Universiti Sains Malaysia; ${ }^{2}$ Universiti \\ Sains Malaysia Hospital, Jalan Raja \\ Perempuan Zainab II, Kubang Kerian, \\ Malaysia
}

\begin{abstract}
Herein, we report our experience in treating extensive traumatic submacular hemorrhage with a single dose of intravitreal ranibizumab. A 23-year-old healthy Malay man presented with a progressive reduction of central vision in the left eye of 2 days' duration following a history of blunt trauma. Visual acuity was reduced to counting fingers. Examination revealed infero-temporal subconjunctival hemorrhage, traumatic anterior uveitis, and an extensive submacular hemorrhage with suspicion of a choroidal rupture in the affected eye. He was initially treated conservatively with topical prednisolone acetate $1 \%$. The subconjunctival hemorrhage and anterior uveitis resolved but his vision remained poor with minimal resolution of the submacular hemorrhage at 1 week follow-up (day 12 post-trauma). In view of the poor resolution of submacular hemorrhage, he was treated with a single dose of $0.5 \mathrm{mg}$ intravitreal ranibizumab at day 20 post-trauma. At 4 weeks post-intravitreal ranibizumab, there was an improvement in visual acuity (from counting fingers to 6/45) and complete resolution of the submacular hemorrhage with presence of a choroidal rupture scar temporal to the fovea, which was not seen clearly at presentation due to obscuration by blood. His visual acuity further improved to $6 / 18$ at 3 months post-trauma. Although this single case had a favorable outcome, a large population cohort study is needed to establish the effectiveness of intravitreal ranibizumab in treating extensive traumatic submacular hemorrhage.
\end{abstract}

Keywords: trauma, choroidal rupture scar, visual acuity, submacular hemorrhage, anterior uveitis

\section{Introduction}

Submacular hemorrhages can be caused by several conditions such as trauma in all age groups and age-related macular degeneration (ARMD), polypoidal choroidal vasculopathy (PCV), and macroaneurysms in the elderly. ${ }^{1}$ Unfortunately, any submacular hemorrhage will result in devastating visual loss if inappropriately managed. The important prognosis factors for vision recovery in traumatic submacular hemorrhage depend on the nature of the injury, length of time before treatment is sought, area of the retina involved, and pre-existing macular function. Damage to the retina can occur within 24 hours and the opportunity for successful recovery is within 2 weeks of onset. ${ }^{2,3}$

The management of traumatic submacular hemorrhage has evolved tremendously from traditional submacular surgery with large retinotomy to a safer pneumatic displacement procedure with or without a tissue plasminogen activator (tPA). ${ }^{3}$ The success of anti-vascular endothelial growth factor (VEGF) in treating submacular hemorrhage related to ARMD, proliferative retinopathy, and PCV is well established, but, as far as 
we are aware, there are no reported studies regarding its usage in traumatic submacular hemorrhage.

Submacular surgery and pneumatic displacement with the use of tPA injection are not without potential complications. Retinal detachment, retinal toxicity, and recurrent hemorrhage do occur. ${ }^{4}$ The success of submacular surgery and pneumatic displacement with gas tamponade are directly related to the duration of the interval between trauma onset and time of seeking treatment because the surgical procedure is only applicable if the patient seeks treatment within 2 weeks of the trauma involving submacular liquefied blood. ${ }^{1,2}$

Herein, we report a case of extensive traumatic submacular hemorrhage that was successfully treated with a single dose of intravitreal ranibizumab.

\section{Case report}

A 23-year-old healthy Malay man presented with reduced central vision in his left eye of 2 days' duration following a history of blunt trauma. He alleged that his left eye had accidentally been hit by a racket while playing badminton. Following the trauma, he developed a reduction in his left central vision that progressively worsened and was associated with mild eye pain. However, there were no floaters, flashes of light, or curtain field defects. He had good vision premorbidly in both eyes prior to the incident.

Ocular examination revealed visual acuity in the left eye was counting fingers at 1 meter and 6/6 in the fellow eye. The anterior segment of the left eye showed a presence of an infero-temporal subconjunctival hemorrhage and a mild anterior chamber reaction. There was absence of relative afferent pupillary defect. Left-eye fundus examination revealed an extensive submacular hemorrhage with suspicion of a choroidal rupture at the inferior margin of the submacular hemorrhage (Figure 1). No retinal tear or retinal detachment was seen. The right eye appeared normal on examination. Optical coherence tomography of the left eye showed increased macular thickness with presence of submacular hemorrhage (Figure 2A and B).

He was diagnosed with traumatic subconjunctival hemorrhage, anterior uveitis, and extensive submacular hemorrhage of the left eye, most probably secondary to choroidal rupture following blunt trauma. He was treated with 4-hourly topical prednisolone acetate $1 \%$ in the left eye for the mild traumatic anterior uveitis and treated conservatively for the submacular hemorrhage. At follow-up at 1 week (day 12 post-trauma), there was no improvement in the vision in his left eye. The subconjunctival hemorrhage and anterior uveitis were resolved, but there was minimal resolution of the submacular

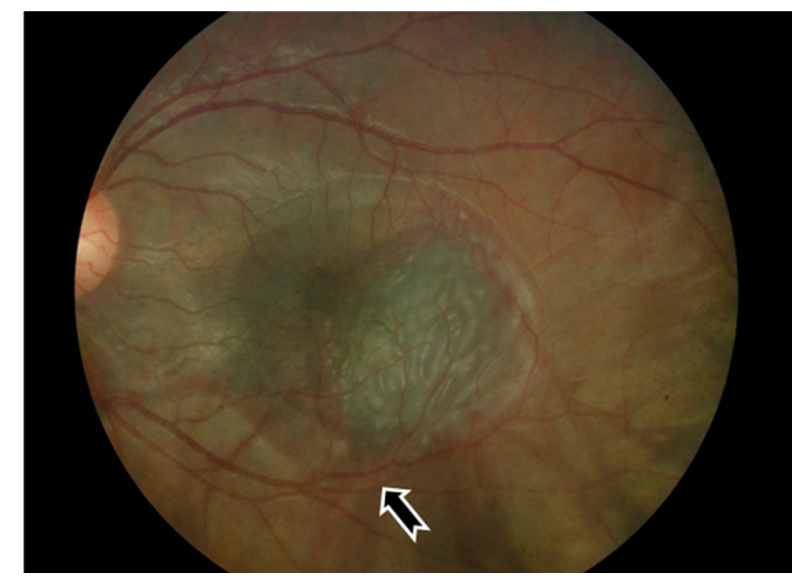

Figure I Left fundus showing extensive submacular hemorrhage with suspicion of choroidal rupture (arrow) at presentation (day 2 post-trauma).

hemorrhage (Figure $2 \mathrm{C}$ and D). The prednisolone acetate $1 \%$ eye drop doses were tapered and then stopped within 2 weeks. In view of the lack of improvement in his vision and poor resolution of the submacular hemorrhage, we decided to trial intravitreal ranibizumab, which was a first for us in treating traumatic submacular hemorrhage.

After discussing various treatment options with the patient and obtaining his informed consent, a single dose of $0.5 \mathrm{mg}$ intravitreal ranibizumab was administered to his left eye using an aseptic technique in a surgical theatre on day 20 following trauma. Four weeks post-intravitreal ranibizumab, his left visual acuity was found to have improved from counting fingers to $6 / 45$. Fundus examination revealed a complete resolution of the submacular hemorrhage with fibrosis scar. A choroidal rupture scar temporal to the fovea was observed, which had not been seen at presentation due to obscuration by blood (Figure 3). Repeat optical coherence tomography showed a reduction in macular thickness and resolution of the submacular hemorrhage with restoration of anatomical retinal architecture (Figure 2E and F). By 3 months following the trauma, the patient's left visual acuity was found to have further improved to $6 / 18$.

\section{Discussion}

Several factors contribute to poor anatomical as well as functional visual recovery in traumatic submacular hemorrhage. These include mechanical damage to the photoreceptors by fibrin infiltration, shearing of photoreceptor cells, iron and hemosiderin toxicity, impaired nutrient supply to the photoreceptors, and fibrotic macular scarring. ${ }^{2,3}$ This indicates that the faster the blood clot resolves, the better anatomical and functional visual recovery is likely to be, since retinal degeneration over areas of dense fibrin occurs within 3-14 days. ${ }^{5}$ 

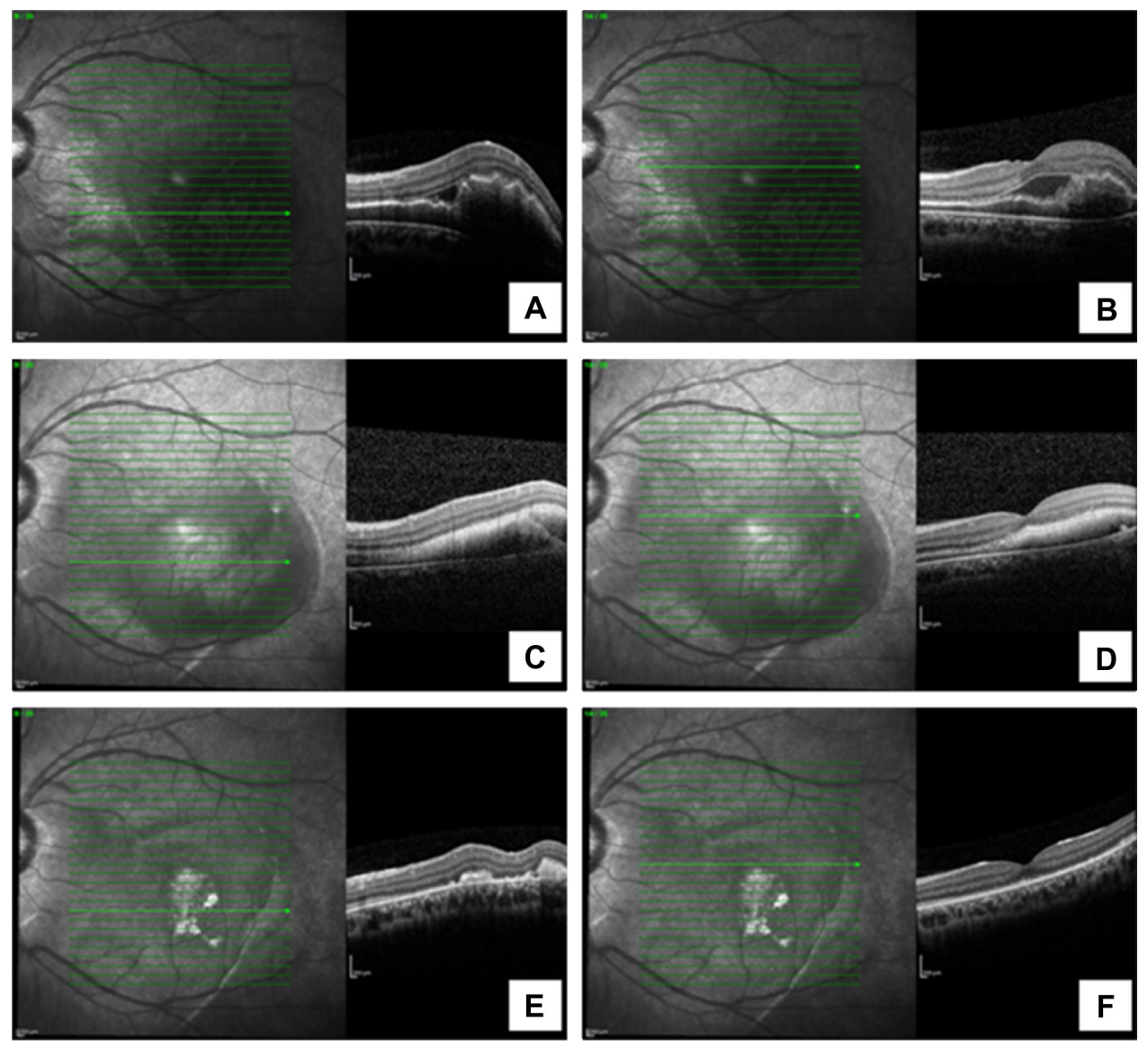

Figure 2 Optical coherence tomography of the left eye showing increased macular thickness with presence of submacular hemorrhage at presentation (A and B), minimal reduction of submacular hemorrhage at I week follow-up (C and D), and resolved submacular hemorrhage with restoration of anatomical retinal architecture at 4 weeks post-intravitreal ranibizumab (E and $\mathbf{F})$.

There is no formal consensus on the management of submacular hemorrhage. In our case, which involved a young man with a premorbidly healthy retina, several treatments could have been offered to him. Submacular surgery was one of the options, but it has devastating complications related to its surgical technique, such as removing retinal pigment epithelium (RPE) and photoreceptor layers together with the blood clot. ${ }^{2}$ Further, the anatomical uncertainty due to the obscuration caused by the hemorrhage can make the surgical procedure more difficult and unpredictable. The Submacular Surgery Trial, ${ }^{6}$ which was undertaken in age-related macular hemorrhage patients, also reported that subretinal extraction of hemorrhage produced equivocal results in comparison to the observation group.

Pneumatic displacement with or without tPA may have been a better option, but this treatment also has certain limitations. Improper pneumatic displacement can lead to shearing of the photoreceptor layers, which will worsen final vision outcome. ${ }^{7,8}$ In addition, the penetration of tPA into the sub-RPE space is poor compared with that of ranibizumab, which indirectly compromises the effect of tPA in deeper subretinal hemorrhage. ${ }^{7,8}$

Conservative treatment involving just observation could have been an option with our patient. However, it would not have been suitable because of the presence of a wide area of submacular hemorrhage involving the fovea, which can predispose a higher risk of poor visual outcome due to the possibility of dense scarring by fibrin-mediated retinal damage. Further, the age of the patient needed to be considered. When the patient is healthy and young, it is better to offer another treatment modality rather than just simple observation. In our case, we decided to trial intravitreal ranibizumab.

Intravitreal ranibizumab has been successfully used to treat submacular hemorrhage related to retinal vascular diseases such as ARMD, PCV, and proliferative retinopathy. As far as we are aware, there have been no reported cases of traumatic submacular hemorrhage treated with ranibizumab. However, in our case, the ranibizumab rapidly resolved the submacular hemorrhage, restored the patient's retinal architecture, and achieved satisfactory visual recovery. 


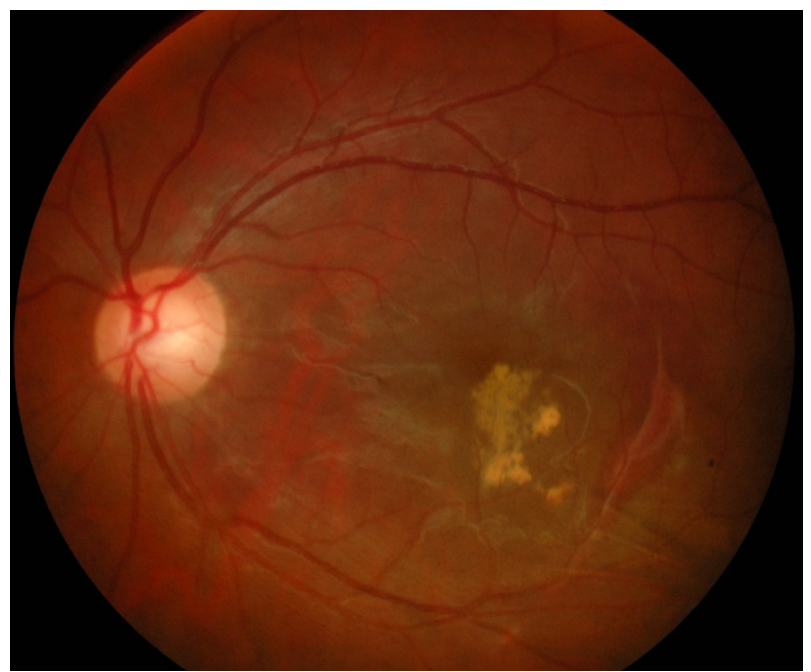

Figure 3 Left fundus showing complete resolution of submacular hemorrhage with fibrosis scar and choroidal rupture scar at 4 weeks post-intravitreal ranibizumab.

The basis of its effectiveness in treating traumatic submacular hemorrhage as in our case is not clearly known. We postulate that the anti-VEGF property as an anti-inflammatory agent played an important role.

Vascular tissue trauma is usually associated with an acute release of large amounts of VEGF as part of the healing process. ${ }^{9}$ This large amount of VEGF may be associated with extensive tissue inflammation due to the high amount of neutrophils released, ${ }^{9}$ which may lead to excessive tissue reaction, delayed healing, and dense scarring. Besides its anti-angiogenesis effect, ranibizumab has also been proven to be a good anti-inflammatory agent, as it modulates inflammatory cells - in particular, fibroblast-related activities. ${ }^{10,11}$ It has demonstrated good penetration into deeper retinal tissues, including the subretinal tissue and sub-RPE space. This may explain its effectiveness in treating conditions related to retinal trauma, including traumatic submacular hemorrhage. ${ }^{4}$ Additionally, ranibizumab has also shown an excellent antiscarring effect in post-trabeculectomy patients by increasing bleb survival rate. ${ }^{10,11}$ However, our hypothesis on the significance of the anti-VEGF property of ranibizumab is just an assumption based on a single case, so further investigation is needed to establish an association.

Clinical Ophthalmology

\section{Publish your work in this journal}

Clinical Ophthalmology is an international, peer-reviewed journal covering all subspecialties within ophthalmology. Key topics include: Optometry; Visual science; Pharmacology and drug therapy in eye diseases; Basic Sciences; Primary and Secondary eye care; Patient Safety and Quality of Care Improvements. This journal is indexed on

\section{Conclusion}

Although a favorable outcome was achieved in this case, a large population cohort study is needed to establish the safety and effectiveness of ranibizumab in treating extensive traumatic submacular hemorrhage. If the results of such a study were favorable, this would enable this treatment to become an alternative or adjuvant therapy to traditional surgery and conventional pneumatic displacement with tPA as a primary treatment in managing traumatic submacular hemorrhage.

\section{Disclosure}

The authors report no conflicts of interest in this work.

\section{References}

1. Hillenkamp J, Surguch V, Framme C, Gabel VP, Sachs HG. Management of submacular hemorrhage with intravitreal versus subretinal injection of recombinant tissue plasminogen activator. Graefes Arch Clin Exp Ophthalmol. 2010;248(1):5-11.

2. Borrillo JL, Regillo CD. Treatment of subretinal hemorrhages with tissue plasminogen activator. Curr Opin Ophthalmol. 2001;12(3):207-211.

3. Sandhu SS, Manvikar S, Steel DH. Displacement of submacular hemorrhage associated with age-related macular degeneration using vitrectomy and submacular tPA injection followed by intravitreal ranibizumab. Clin Ophthalmol. 2010;4:637-642.

4. Treumer F, Klatt C, Roider J, Hillenkamp J. Subretinal coapplication of recombinant tissue plasminogen activator and bevacizumab for neovascular age-related macular degeneration with submacular haemorrhage. Br J Ophthalmol. 2010;94(1):48-53.

5. Toth CA, Benner JD, Hjelmeland LM, Landers MB 3rd, Morse LS. Ultramicrosurgical removal of subretinal hemorrhage in cats. $\mathrm{Am} J$ Ophthalmol. 1992;113(2):175-182.

6. Bressler NM, Bressler SB, Childs AL, et al; Submacular Surgery Trials (SST) Research Group. Surgery for hemorrhagic choroidal neovascular lesions of age-related macular degeneration: ophthalmic findings: SST report no 13. Ophthalmology. 2004;111(11):1993-2006.

7. Kamei M, Misono K, Lewis H. A study of the ability of tissue plasminogen activator to diffuse into the subretinal space after intravitreal injection in rabbits. Am J Ophthalmol. 1999;128(6):739-746.

8. Pulido JS, Bakri SJ, Valyi-Nagy T, Shukla D. Rituximab penetrates full-thickness retina in contrast to tissue plasminogen activator control. Retina. 2007;27(8):1071-1073.

9. Barleon B, Sozzani S, Zhou D, Weich HA, Mantovani A, Marmé D. Migration of human monocytes in response to vascular endothelial growth factor (VEGF) is mediated via the VEGF receptor flt-1. Blood. 1996;87(8):3336-3343.

10. Messmer EM, Zapp DM, Mackert MJ, Thiel M, Kampik A. In vivo confocal microscopy of filtering blebs after trabeculectomy. Arch Ophthalmol. 2006;124(8):1095-1103.

11. Tran J, Rak J, Sheehan C, et al. Marked induction of the IAP family antiapoptotic proteins survivin and XIAP by VEGF in vascular endothelial cells. Biochem Biophys Res Commun. 1999;264(3):781-788.
PubMed Central and CAS, and is the official journal of The Society of Clinical Ophthalmology (SCO). The manuscript management system is completely online and includes a very quick and fair peer-review system, which is all easy to use. Visit http://www.dovepress.com/ testimonials.php to read real quotes from published authors. 